\title{
Application of Soft-Computing Techniques in Modelling of Buildings
}

\section{Azzi, A E Gegov, G S Virk, B P Haynes and K Alkadhimi}

University of Portsmouth, Department of EEE, Anglesea Building, Portsmouth PO1 3DJ, UK, Tel: +44-(0)23-92842763, Fax: +44-(0)23-92842766,

alexander.gegov@port.ac.uk

\begin{abstract}
Summary:
The paper presents recent results on the application of soft computing techniques for predictive modelling in the built sector. More specifically, an air-conditioned zone (Anglesea Building, University of Portsmouth), a naturally ventilated room (Portland Building, University of Portsmouth), and an endothermic building (St Catherine's Lighthouse, Isle of Wight) are considered. The zones are subjected to occupancy effects and external disturbances which are difficult to predict in a quantitative way and hence the soft computing approach seems to be a better alternative. In fact, the overall complexity of the problem domain makes the modelling of the internal climate in buildings a difficult task which is not always carried out in a satisfactory way by traditional deterministic and stochastic methods. The approach adopted uses fuzzy logic for modelling, as well as neural networks for adaptation and genetic algorithms for optimisation of the fuzzy model. The latter is of the Takagi-Sugeno type and it is built by subtractive clustering as a result of which the initial values of the antecedent non-linear membership functions and the consequent linear algebraic equations parameters are determined. A method of a combinatorial search over all possible fuzzy model structures for a specified plant order is presented. The model parameters are further adjusted by a back-propagation neural network and a real-valued genetic algorithm in order to obtain a better fit to the measured data. Modelling results with actual data from the three buildings are presented where the initial (fuzzy) and the final (fuzzy-neuro and fuzzy-genetic) models are shown.
\end{abstract}

Keywords: $\quad$ Soft-computing, intelligent modelling, building management systems.

\section{Introduction}

Soft Computing (SC) is a heuristic methodology which has attracted significant interest in recent years and has shown to be successful in many areas such as modelling, control, fault diagnosis and pattern recognition. It is based on the implementation of different approaches such as Fuzzy Logic (FL), Neural 
Networks (NN), Genetic Algorithms (GA), and some others [5], [6]. Each of these techniques is suited for solving specific types of problems. In this respect, FL is powerful for approximate modelling and reasoning, $\mathrm{NN}$ are well suited for learning-based adaptation while GA are efficient for evolutionary-based optimisation. The underlying idea of SC is to use these heuristic approaches in combination with each other as well as with some conventional approaches, rather than using them separately.

Recent research in the field has shown a steady trend to hybrid solutions incorporating different SC approaches together as well as other classical approaches. A typical example is the so-called Takagi-Sugeno fuzzy models exploiting the idea of conventional dynamic modelling from numerical data embedded into a fuzzy logic inference framework. These models can be further improved by NN or GA to obtain a better fit to the measured data.

However, Takagi-Sugeno models usually assume a structure of the plant which is given a priori rather than trying to find it by some search procedures. In this respect, the paper presents a method of a combinatorial search over all possible model structures for a specified plant order. The method is demonstrated for a fuzzy model which predicts internal temperature and relative humidity values in three different buildings but can be applied to other types of plants as well.

\section{Predictive Modelling Based Control}

The work proposed here is concerned with the efficient control of the internal climate in office buildings. The authors' aim is to develop good predictive models which will allow a proactive control policy to be adopted rather than the traditional reactive ones in current use. In other words, instead of applying a control action only on the basis of the current sensor readings, it is also necessary to predict the values over a certain time interval. The main advantage of this proactive philosophy lies in the possibility to apply heating and cooling control efforts more efficiently as a result of which the control becomes smoother, together with smaller overshoots and shorter settling times. This, in turn, leads to decreased energy consumption and reduced pollution of the environment. However, to obtain predictive models for these buildings is not an easy task because their performance is affected by climatic and occupancy effects which are characterised by significant complexity and uncertainty.

The notion of a proactive control philosophy is the following. The control action at the current time instant $k$ is computed not only on the basis of the measured output at the same time instant and the previous ones $(k-1),(k-2)$, etc, but also by taking into account the predicted values of the output at the future time instants $(k+1),(k+2)$, etc.

It would be interesting to see, if the SC methodology can provide good models for Building Management Systems (BMS). Some investigations have recently been 
carried out in this domain but most of them are too narrow and are thus leading to limited conclusions. In most cases, they are focused on one modelled parameter in one building during one season, and make use of separate SC techniques, rather than adopt a coordinated SC methodology. In other words, the potential of SC methodology for BMS has still not been explored in any detail.

This paper presents recent results from a research project aimed at investigating in a systematic way the capabilities of the SC methodology for predictive modelling of internal parameters in buildings, namely temperature and relative humidity [1], [2], [3], [4]. In this respect, air conditioned, naturally ventilated and endothermic types of buildings are considered. The three specific buildings under investigation are: Anglesea Building (University of Portsmouth), Portland Building (University of Porsmouth) and St Catherine's Lighthouse (Isle of Wight). These types of buildings are widely used nowadays and it must be pointed out that they differ substantially in their mode of functioning. Therefore, it is intended to find how the $\mathrm{SC}$ methodology is suited to each type of building.

\section{Data Based Fuzzy Modelling}

The approach adopted is based on a Takagi-Sugeno (TS) fuzzy model which has received considerable attention recently because of its suitability for processing information from input-output measurements. This is the case in BMS where the main information source is numerical data from sensor readings rather than expert knowledge which is difficult to obtain because of the multivariable and coupled nature of the process [2], [5]. Another advantage of the TS fuzzy model is its capability to approximate non-linear input-output mappings by a number of locally linearised models.

The TS fuzzy model consists of linguistic if-then rules in the antecedent part and linear algebraic equations in the consequent part. There are two types of parameters in this model: non-linear (in the membership functions in the antecedent part) and linear (in the algebraic equations in the consequent part). The task of the fuzzy model is to determine the initial values of both types of parameters on the basis of the input-output data. The method used in the paper is based on the idea of subtractive clustering, i.e. by assuming that each data point is a potential cluster centre and gradually finding the final clustering.

The Takagi-Sugeno fuzzy model for a system with two rules, two inputs $\left(u_{1}, u_{2}\right)$ and one output $(y)$ is presented by Equation (1). The linguistic labels (membership functions) of the inputs are denoted by $A_{i}, B_{i}, i=1,2$ and their parameters are the non-linear antecedent parameters. The coefficients $a_{i}, b_{i}$, $i=1,2,3$ are the linear consequent parameters used for the computation of the output.

If $u_{1}$ is $A_{1}$ and $u_{2}$ is $A_{2}$ then $y=a_{1} \cdot u_{1}+a_{2} \cdot u_{2}+a_{3}$

if $u_{1}$ is $B_{1}$ and $u_{2}$ is $B_{2}$ then $y=b_{1} \cdot u_{1}+b_{2} \cdot u_{2}+b_{3}$ 
Equation (1) represents a static Takagi-Sugeno fuzzy model which does not contain the time argument in the input and the output variables. However, in order to predict future temperatures, the time argument needs to be included in the equation, i.e. the model must be a dynamic one. In this respect, two types of dynamic models are introduced and investigated in the paper, namely Regression Delay (RD) and Proportional Difference (PD). Examples of such models are represented by Equations (2) and (3), respectively.

If $y_{k-1}$ is $A_{1}$ and $y_{k-2}$ is $A_{2}$ and $u_{l, k-1}$ is $A_{3}$ and $u_{l, k-2}$ is $A_{4}$ and $u_{2, k-2}$ is $A_{5}$

then $y_{k}=a_{1} \cdot y_{k-1}+a_{2} \cdot y_{k-2}+a_{3} \cdot u_{1, k-1}+a_{4} \cdot u_{1, k-2}+a_{5} \cdot u_{2, k-2}+a_{6}$

If $y_{k-1}$ is $A_{1}$ and $D y_{k-1}$ is $A_{2}$ and $u_{1, k-1}$ is $A_{3}$ and $D u_{2, k-1}$ is $A_{4}$

then $y_{k}=a_{1} \cdot y_{k-1}+a_{2} \cdot D y_{k-2}+a_{3} \cdot u_{l, k-1}+a_{4 \cdot} \cdot D u_{2, k-l}+a_{5}$

where $D y_{k-I}=y_{k-I}-y_{k-2}, D u_{2, k-1}=u_{2, k-I}-u_{2, k-2}$

It can be seen that Equation (2) contains two auto-regressive terms of the output $y$, two regressive terms of the input $u_{1}$ and one delay term of the input $u_{2}$. As opposed to this, Equation (3) contains one proportional and one derivative term of the output $y$, one proportional term of the input $u_{l}$ and one derivative term of the input $u_{2}$. For simplicity, each of the equations includes only one rule, but in general the number of rules is higher. More specifically, it is equal to the number of the linearised submodels applicable to the respective local regions of the whole operating range.

Equations (2)-(3) represent examples of fuzzy model structures. Usually, these structures are obtained on the basis of evaluation of a number of structures in accordance with a performance criterion that is usually the Root Mean Squared Error (RMSE). The latter is a measure of the closeness of the model to the plant.

However, the majority of identification techniques apply partial rather than extensive model structural searching. They tend to represent the plant dynamics by either regression or delayed terms. As far as proportional or difference terms are concerned, they are usually considered up to the most recent data point in the past. This might be a serious disadvantage as the dynamics of the plant are not fully explored and some important terms in the model structure might be missed.

This paper presents a method of extensive searching of the model structures to explore the possible dynamics of the plant for a specified order. This method is demonstrated here for Takagi-Sugeno fuzzy models but can be also applied to any other types of models, including traditional ones. In this case, for a plant with $m$ input variables and backward horizon equal to $b$, the whole number of investigated models is given by the equation:

$C=(2 . b)^{m}-1$ 
It is evident from Equation (4) that the number of investigated models is an exponential function of the number of inputs and the backward horizon which leads to a considerable increase of the computational complexity as the order grows. This is the price to pay for the extensive searching.

\section{Neural Adaptation and Genetic Optimisation}

The task of neural adaptation is to adjust the model parameters in order to obtain a better fit to the measured data. The method used in the paper is based on the idea of back-propagation, i.e. by iterative propagating of the model error (the difference between the real and the modelled plant output) from the consequent to the antecedent part of the fuzzy rules until a specified number of iterations is reached.

The neural adaptation algorithm involves the following steps:

1. Fix the antecedent parameters $A_{i}$ in the non-linear membership functions.

2. Estimate the consequent parameters $a_{i}$ by a least squares procedure.

3. Compute the model output $y_{k}$.

4. Keep the consequent parameters $a_{i}$ in the linear equations fixed.

5. Estimate the antecedent parameters $A_{i}$ by a gradient descent procedure.

6. Compute the RMSE of the model.

7. Go to step 1 if the specified number of iterations has not been reached.

8. Stop.

The purpose of genetic optimisation is also to adjust the model parameters as an alternative to the neural adaptation. The method used in the paper is based on the idea of real-valued coding, i.e. by representing the individuals with real valued genes and sequential evaluation of the model error until a pre-specified number of generations is reached.

The genetic optimisation algorithm involves the following steps:

1. Define the variation ranges for the antecedent and the consequent parameters $A_{i}$ and $a_{i}$.

2. Create initial generation of individuals containing the parameters $A_{i}$ and $a_{i}$ as genes.

3. Evaluate the fitness function of all individuals by the RMSE of the model.

4. Select the fitter half of individuals for crossover.

5. Apply crossover on these individuals and create new ones.

6. Replace the less fit half of individuals with the new ones.

7. Apply mutations on some individuals

8. Evaluate the fitness function of all individuals by the RMSE.

9. Go to step 4 if the specified number of generations has not been reached.

10. Stop. 


\section{Experimental Results}

This section presents modelling results from the three buildings under study. The results show that the outputs (temperature and relative humidity) can be predicted to a high level of accuracy on the basis of measurements. The most important variables used in the modelling are chosen by carrying out a cross-correlation analysis involving temperatures and relative humidities in the same and neighbouring zones, as well as external (weather) variables, e.g. solar radiance, wind speed and direction, etc. To ensure systematic modelling, both regressiondelay and proportional-difference types of fuzzy model structures have been considered. The best model structure corresponding to the smallest training (identification) error was chosen by evaluating all possible structures of models up to some specified order, i.e. involving all combinations of input terms. Afterwards, the quality of the models was evaluated with new data and the validation errors obtained to give a measure of the prediction accuracy of these models. The validation errors are the difference between the model predictions and the actual outputs. The membership functions of the inputs in the fuzzy model were chosen to be of the Gaussian type while the selected options for the neural network and the genetic algorithm were 500 iterations and 50 generations with 10 individuals each, respectively. These options seem to give a good comparison between the two model improvement techniques used in our work.

Each one of the above model structures was chosen from a set of 1023 possible models, representing all the combinations of (auto)regression and (auto)delay terms. The backward (dynamic memory) horizon was chosen equal to 2, i.e., the model predictions at time $k$ are obtained on the basis of measurements at times $k-1$ and $k-2$. The plant and model outputs for each of these models and for each building are shown in Figures 1-3.

\section{Conclusions}

Both the neural adaptation and the genetic optimisation schemes lead to a substantial improvement of the prediction capabilities of fuzzy model that have been formulated using traditional TS methods. These methods can be improved by incorporating a model structure selection capacity. The improvement can be considerable, especially in the cases where the model is not very good. The quality of the temperature prediction is better than the humidity prediction for all three buildings because of the smaller variational range and smoother profile.

The proportional-difference fuzzy model performs better than the regression-delay model in most cases for all the buildings indicating that it can capture the dynamics of the buildings more precisely. Concerning the model improvement, using genetic algorithms is in most cases superior to using neural networks for both temperature and relative humidity, for both types of regression-delay and proportional-difference fuzzy models, as well as for all three buildings. It has to be 

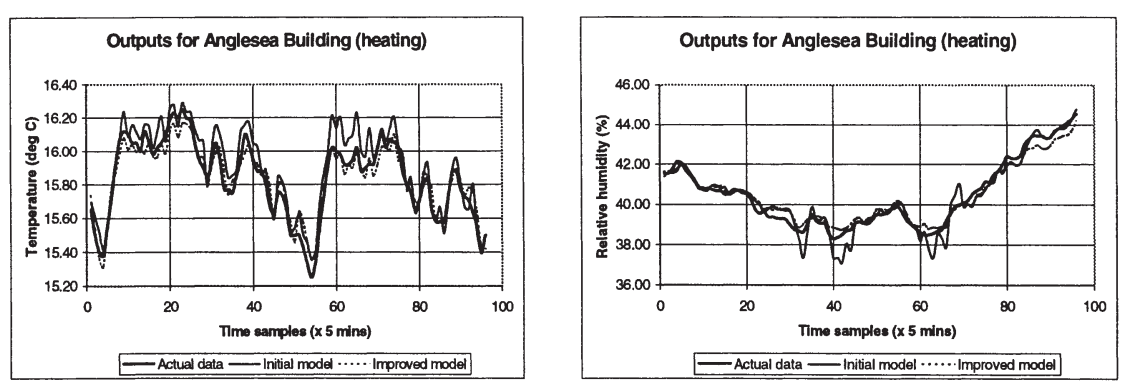

Figure 1. Temperature and relative humidity for Anglesea Building.
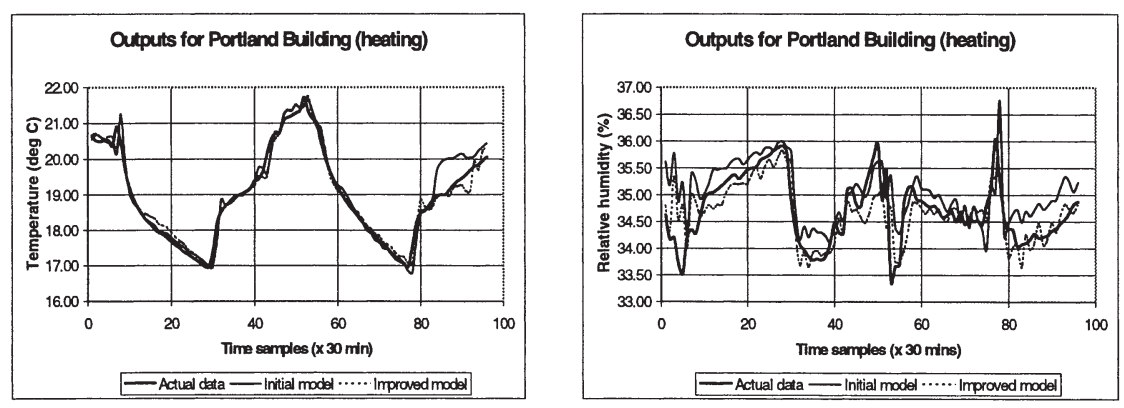

Figure 2. Temperature and relative humidity for Portland Building.
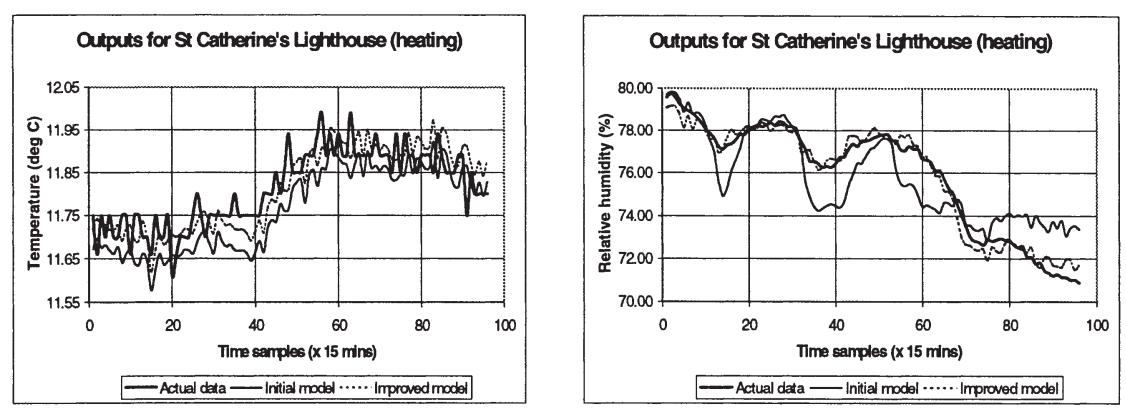

Figure 3. Temperature and relative humidity for St Catherine's Lighthouse. 
noted that the best prediction results have been obtained for the naturally ventilated room, while the results for the air-conditioned zone and the ambient energy building are not as good but are comparable to each other.

With respect to the comparison between the two model improvement techniques, genetic algorithms seem to be superior. They show considerably better convergence properties and the best model parameters are usually obtained from the last genetic generations. In contrast, when using neural networks, the best model parameters are in most cases found from the first neural iterations. Taking also into account the parallel search capabilities of genetic algorithms, it is possible to conclude that they offer a far more reliable approach for improving fuzzy models than neural networks.

\section{Acknowledgement}

This work was carried out within an EPSRC funded project 'Soft Computing Models for Building Applications - a Feasibility Study' (Ref: GR/L84513). This funding is gratefully acknowledged. In addition, the project involves collaboration with Satchwell Control Systems, Caradon Trend Ltd, Ambient Energy Systems Ltd, Hampshire County Council, the Building Research Establishment and Trinity House Lighthouse Service. The technical and financial support provided by these partners is also greatly appreciated.

\section{References}

[1] Gegov, A, Virk, G, Azzi, D, Haynes, B and Alkadhimi, K, "Soft-computing based predictive modelling of building management systems", International Workshop on Recent Advances in Soft Computing, Leicester, UK, 69-77, 1999.

[2] Gegov, A, Virk, G, Azzi, D, Haynes, B and Alkadhimi, K, "Soft-computing based modelling of the internal climate in office buildings", UK Workshop on Fuzzy Systems, Uxbridge, UK, 145-152, 1999.

[3] Gegov, A, Virk, G, Azzi, D, Haynes, B, Alkadhimi, K and Matthews, I, "Neuro-fuzzy adaptive modelling of air-conditioning systems", European Congress on Intelligent Techniques and Soft Computing, Aachen, Germany, 267268, 1999.

[4] Gegov, A, Virk, G, Azzi, D, Haynes, B, Alkadhimi, K, "Soft computing models of naturally ventilated buildings", CIBSE National Conference, London, UK, 421-428, 1999.

[5] Haupt, R and Haupt, S, "Practical Genetic Algorithms", John Wiley \& Sons, New York, 1998.

[6] Jang, J, Sun, C and Mizutani, E, "Neuro-Fuzzy and Soft Computing", Prentice Hall, Upper Saddle River, 1997. 